\title{
Effect of metformin on residual cells after chemotherapy in a human lung adenocarcinoma cell line
}

\author{
SATORU KITAZONO $^{1}$, YUICHI TAKIGUCHI ${ }^{2}$, HIRONORI ASHINUMA ${ }^{1}$, MIYAKO SAITO-KITAZONO ${ }^{1}$, \\ ATSUSHI KITAMURA ${ }^{1}$, TETSUHIRO CHIBA ${ }^{3}$, EMIKO SAKAIDA ${ }^{2}$, IKUO SEKINE $^{2}$, \\ YUJI TADA $^{1}$, KATSUSHI KUROSU ${ }^{1}$, SEIICHIRO SAKAO ${ }^{1}$, NOBUHIRO TANABE ${ }^{1}$, \\ ATSUSHI IWAMA ${ }^{4}$, OSAMU YOKOSUKA ${ }^{3}$ and KOICHIRO TATSUMI ${ }^{1}$
}

Departments of ${ }^{1}$ Respirology, ${ }^{2}$ Medical Oncology, ${ }^{3}$ Medicine and Clinical Oncology, and ${ }^{4}$ Cellular and Molecular Medicine, Graduate School of Medicine, Chiba University, Chuo-ku, Chiba 260-8670, Japan

Received August 5, 2013; Accepted September 16, 2013

DOI: 10.3892/ijo.2013.2120

\begin{abstract}
Cancer chemotherapy, including molecular targeted therapy, has major limitations because it does not kill all the cancer cells; the residual cells survive until they acquire chemoresistance. In the present study, the combined effects of metformin and gefitinib were examined in vivo in a mouse xenograft model, inoculated with a human lung adenocarcinoma cell line that possesses an activating epidermal growth factor receptor mutation. The mechanism of the interaction was further elucidated in vitro. Metformin did not suppress the growth of already established tumors, nor did metformin augment tumor shrinkage by gefitinib. However, metformin significantly suppressed the regrowth of the tumor after effective treatment with gefitinib, suggesting the specific effect of metformin on the residual cells. Cytotoxicity of metformin was characterized by the absence of apoptosis induction and unremarkable cell cycle shift in vitro. The residual cell population after treatment with gefitinib was characterized by enriched cells with high expression of CD133 and CD24. Metformin was still effective on this specific cell population. Targeting residual cells after chemotherapy may represent an effective novel strategy for the treatment of cancer. Elucidating the mechanism of metformin cytotoxicity provides insights into future development of anticancer therapeutics.
\end{abstract}

\section{Introduction}

Cytotoxic chemotherapy of most solid cancers rarely cures the cancer. Molecular targeted therapy has the same limitation, despite significantly enhanced cancer control and resulting

Correspondence to: Professor Yuichi Takiguchi, Department of Medical Oncology, Graduate School of Medicine, Chiba University, 1-8-1 Inohana, Chuo-ku, Chiba 260-8670, Japan

E-mail: takiguchi@faculty.chiba-u.jp

Key words: residual cell, chemoresistance, metformin, lung cancer, gefitinib prolonged survival, especially when cancers with driver mutations are treated with specific inhibitors, for example, when non-small cell lung cancer with an epidermal growth factorreceptor $(E G F R)$ mutation is treated by EGFR-tyrosine kinase inhibitors (TKIs) (1). Thus, even after a complete response after a long period of treatment with gefitinib, the tumors grew again after withdrawal of the agent in an animal study (2). The secondary point mutation of T790M in EGFR (3) and establishment of a bypass signal transduction via $c M E T$ amplification (4) are known genetic alterations responsible for acquired EGFR-TKI resistance. If chemotherapy were sufficiently effective to kill the entire cell population of the tumor in a short period, however, the cancer would be cured before genetic adaptation and chemoresistance. Nevertheless, some cells in the tumor escape from effective chemotherapy and these residual cells survive until a chemoresistant phenotype is obtained. Therefore, complete elimination of residual cells would represent significant progress in cancer therapy. A recent study showed that the drug-tolerant phenotype, induced by acute response to chemotherapeutic agents, is reversible and that the phenotype maintains viability via engagement of insulin-like growth factor (IGF)-1 receptor signaling and an altered chromatin state that requires histone demethylase (5). This observation importantly provides a vision for a new strategy to treat cancer by specifically targeting the residual cells after chemotherapy.

Metformin is a safe biguanide that has been used worldwide to treat type 2 diabetes mellitus. Metformin activates AMP-activated protein kinase (AMPK), an enzyme that plays an important role in insulin signaling, whole body energy balance and the metabolism of glucose and fats, resulting in lowering of blood glucose (6). Metformin recently attracted attention for its potential anticancer effects (7).Epidemiological studies (8-10) first suggested a link between metformin and cancer prevention by demonstrating a lower incidence of death from cancer in patients with diabetes mellitus treated with metformin than those treated with other antidiabetic agents. These studies were followed by clinical observations, suggesting a link between metformin and increased pathologically complete response rate by induction chemotherapy in patients with breast cancer (10) as well as lower incidence rate 
of metastasis and a reduced risk of death in patients with lung cancer (11). These findings triggered a number of in vitro and in vivo experiments, revealing its antiproliferative properties in a variety of cancers (12-20). Although the precise mechanism is unclear, activation of AMPK might be crucial. First, liver kinase B1 (LKB1), a well-recognized tumor suppressor, activates AMPK $(21,22)$ and metformin requires LKB1 for growth inhibitory action (23). Second, AMPK inhibits the mammalian target of rapamycin (mTOR) and the S6 kinase I pathways $(24,25)$ and this inhibition appears to be achieved by phosphorylating tuberous sclerosis complex-2, another tumor suppressor and upstream regulator of mTOR (26). Notably, metformin blocks the growth-promoting effects of both insulin and IGF-1, deregulates AMPK activity and inhibits mTOR activity, S6 kinase activity and protein synthesis both in transformed and non-transformed mammary gland cells (14). However, it is unknown whether metformin causes apoptosis of cancer cells $(13,17)$ or not $(12,16)$, or whether metformin kills cancer cells synergistically with other cytotoxic agents $(15,18,20,27)$ or antagonistically to cisplatin $(28,29)$.

In the present study, in vivo experiments suggested a unique anticancer action for metformin, specifically on residual cells after chemotherapy. The mechanism was further elucidated with a series of in vitro experiments.

\section{Materials and methods}

Cell culture and reagents. A human lung adenocarcinoma cell line, PC9, purchased from Riken Cell Bank (accession no. RCB4455, Tsukuba, Japan), was used throughout the study. This cell line has an activating deletion of the EGFR gene (del E746-A750) in exon 19 (30). The cells were cultured as a monolayer in RPMI-1640 medium supplemented with $10 \%$ fetal bovine serum (FBS), $100 \mathrm{U} / \mathrm{ml}$ penicillin and $100 \mathrm{mg} /$ $\mathrm{ml}$ streptomycin in a $37^{\circ} \mathrm{C}$ humidified atmosphere containing $5 \% \mathrm{CO}_{2}$. Gefitinib (cat no. 3000, Tocris Bioscience, Ellisville, MO, USA) was dissolved in dimethyl sulfoxide (DMSO) and stored at $-20^{\circ} \mathrm{C}$ until use. Metformin (1,1-dimethylbiguanide hydrochloride, cat no. D150959-5G, Sigma-Aldrich, St. Louis, MO, USA) was dissolved in phosphate buffered saline (PBS) at a concentration of $100 \mathrm{mM}$ and stored at $4^{\circ} \mathrm{C}$. A cisplatin solution at a concentration of $0.5 \mathrm{mg} / \mathrm{ml}(\mathrm{pH} 2.5-5.5)$ was purchased from Nihon Kayaku (Tokyo, Japan). Each drug was diluted in the complete medium for each experiment and the final concentration of DMSO was $<0.1 \%$.

Combined treatment of metformin and gefitinib in a mouse xenograft model. Five to 6-week-old female severe combined immunodeficient (SCID) mice were acclimatized to local conditions for a week before starting the experiments. Aliquots of the cell suspension $\left(2 \times 10^{6}\right.$ cells per mouse) were injected subcutaneously into their flanks. At day 16 (when the tumor volumes had reached $\sim 300 \mathrm{~mm}^{3}$ ), the mice were randomly allocated into 4 groups ( 7 mice per group). In every group, administration of either saline alone or gefitinib suspended in saline (150 mg/kg/day, every day, p.o. with gavage) and either PBS alone or metformin dissolved in PBS $(250 \mathrm{mg} / \mathrm{kg} / \mathrm{day}$, every day, i.p.) were started. Either saline alone or gefitinib suspended in saline was continued for 14 days and either PBS alone or metformin dissolved in PBS was continued until terminating observation. In the first group, only saline (p.o.) and PBS (i.p.) were administered (control). In the second group, metformin dissolved in PBS was administered. In the third group, gefitinib suspended in saline was administered. In the fourth group, both gefitinib and metformin were administered (Fig. 1). The administration route of metformin was selected because a previous study showed that i.p. was better tolerated and more effective than p.o. in vivo (31). The dose of metformin was selected according to a preliminary experiment that showed that this dose was near maximal without causing death or body-weight loss in the animals (data not shown). The dose of gefitinib was previously published (32). In each animal, the tumor was allowed to grow until reaching $\sim 2,000 \mathrm{~mm}^{3}$, or until day 66 , after which the animal was sacrificed. The tumor size was estimated by 2-dimensional caliper measurements and calculation with the formula $\pi / 6 \times(\mathrm{A} \times \mathrm{B})^{3 / 2}$, where $\mathrm{A}$ and $\mathrm{B}$ represent the larger and smaller diameters of the tumor, respectively. The tumor size was measured twice a week during the observation period. The animal experiments were approved by the animal ethics review board of Chiba University (protocol no. A22-186) and were conducted in an animal facility at Chiba University under the strict SPF conditions in accordance with the established institutional guidelines.

Cell proliferation in vitro. For the in vitro chemosensitivity assay, $5 \times 10^{5}$ cells per $6-\mathrm{cm}$-diameter culture dish were plated and cultured for $24 \mathrm{~h}$ until adding the agents metformin, gefitinib, cisplatin, or their combinations, to the medium for further culture. The cells were harvested, counted and the survival at defined time points (24, 48 and $72 \mathrm{~h}$ after adding the agents) was calculated. A series of preliminary experiments indicated that metformin at $10 \mathrm{mM}$, gefitinib at $0.03 \mu \mathrm{M}$ and cisplatin at $1.5 \mu \mathrm{g} / \mathrm{ml}$ were nearly equivalent in reducing cell numbers to $10 \%$ of the cell number obtained without adding the agents (control) at $72 \mathrm{~h}$ (Fig. 2). Therefore, these concentrations were used for further experiments.

Apoptosis. Apoptosis was evaluated by Hoechst staining and caspase activity determination. For Hoechst staining, cells treated with agents for $48 \mathrm{~h}$ were trypsinized and harvested together with the floating cells, fixed with $70 \%$ ethanol, stained with $2 \mu \mathrm{g} / \mathrm{ml}$ bisbenzimide $\mathrm{H} 33342$ trihydrochloride (cat no. B2261-25MG; Sigma-Aldrich) and examined by fluorescence microscopy according to the manufacturer's instructions. Cells with aggregated or fragmented chromatin were regarded as apoptotic cells and 500 cells in each experiment were counted to calculate the apoptotic cell ratio. To determine the caspase activity in the cell extracts, a colorimetric assay was used to monitor the absorbance at $405 \mathrm{~nm}$ of $p$-nitroanilide ( $p \mathrm{NA}$ ) released from the synthetic substrates. Caspase 3 and 8 activities were evaluated with synthetic substrates DEVD-pNA and IETD-pNA, respectively, using the colorimetric assay kit APOPCYTO (Medical \& Biological Laboratories, Nagoya, Japan) according to the manufacturer's instructions. In each assay, $\sim 200 \mu \mathrm{g}$ of protein was extracted from the cells treated with each agent for $24 \mathrm{~h}$.

Cell cycle. Cell cycle distributions were determined by a propidium iodide single-color flow cytometric method 


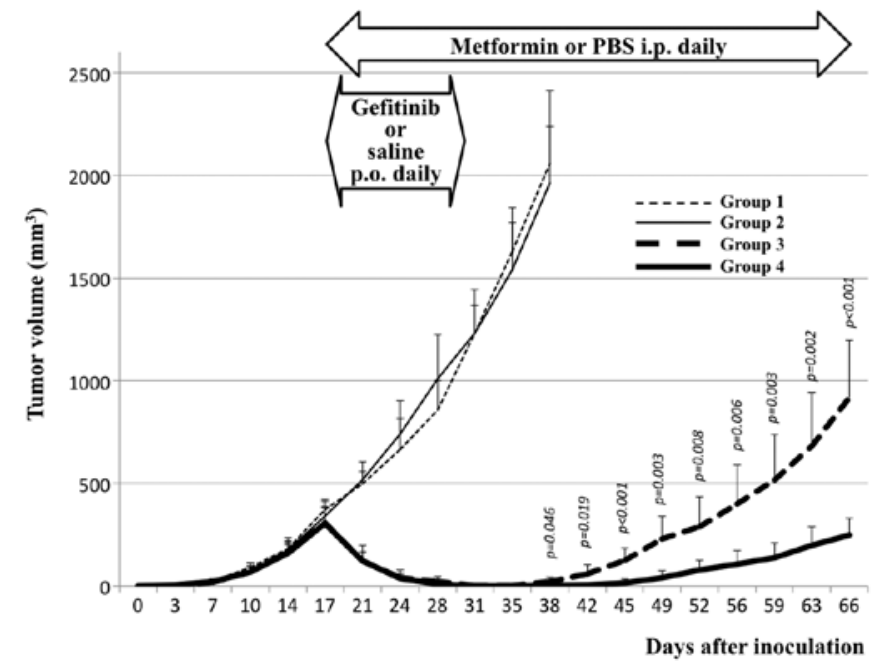

Figure 1. Effects of treatment with metformin, gefitinib and a combination of metformin and gefitinib, on the growth of PC9 xenograft tumors in SCID mice. After growing tumors for 16 days, the animals were randomly divided into 4 groups. In group 1 (fine broken line), saline (p.o.) was administered daily for 14 days (until day 30) and PBS (i.p.) was then administered daily until terminating the observation (day 66). In group 2 (fine, solid line), gefitinib (p.o., $150 \mathrm{mg} / \mathrm{kg} /$ day) suspended in saline was administered daily for 14 days and PBS (i.p.) was then administered daily until day 66. In group 3 (thick, broken line), saline (p.o.) was administered daily for 14 days and metformin (i.p., $250 \mathrm{mg} / \mathrm{kg} /$ day) dissolved in PBS was then administered daily until day 66. In group 4 (thick, solid line), both gefitinib and metformin were administered. The regrowth of the tumors after withdrawing gefitinib was significant, with each given p-value in the figure, suppressed by metformin (compare groups 3 and 4), whereas metformin exerted no effects on tumor growth (compare groups 1 and 2) and tumor shrinkage by gefitinib (compare groups 3 and 4). Each point represents the mean and the bars represent the $\operatorname{SE}(n=7)$.

(FACSCanto II; BD Biosciences, San Jose, CA, USA), according to the manufacturer's instructions. Briefly, the cells were trypsinized, washed twice with ice-cold PBS, fixed with $70 \%$ ethanol and then stored at $-20^{\circ} \mathrm{C}$ until analysis. Before analysis by FACS and CellQuest software (BD Biosciences), the cell suspensions were washed twice with PBS, suspended in $500 \mu \mathrm{l}$ of PI/RNase staining buffer (BD Biosciences) and incubated for $15 \mathrm{~min}$ at room temperature.

Immunofluorescent staining for CD133. The cells were cultured in a chamber slide for $24 \mathrm{~h}$, followed by treatment with the agents for $24 \mathrm{~h}$. After removal of the medium containing the agents, the cells were fixed in a 1:1 mixture of methanol and acetone for $2 \mathrm{~min}$, followed by blocking with normal goat serum for $30 \mathrm{~min}$. The cells were incubated with primary anti-human CD133 antibodies (cat no. 130-090-422, Miltenyi Biotec, Bergisch Gladbach, Germany) overnight at $4^{\circ} \mathrm{C}$. The cells were then washed 3 times in PBS and incubated with the secondary antibody (anti-mouse $\mathrm{IgG}$ ) conjugated to the Alexa488 fluorescent dye for $1 \mathrm{~h}$ at room temperature. The stained cells were embedded in VectaShield mounting medium with DAPI (Vector Laboratories, Burlingame, CA, USA) and were examined with a Nikon Eclipse 80i microscope (Nikon, Tokyo, Japan) using the VB-7210 imaging system (Keyence, Tokyo, Japan). Staining results were directly observed at a magnification of $\mathrm{x} 200$ using a fluorescence microscope.

CD24 and CD44 expression determined by FACS. For analysis of cell-surface marker expression by FACS, anti-human CD24 antibodies conjugated to phycoerythrin (cat no. 311105, BioLegend, San Diego, CA, USA) and anti-human CD44 antibodies conjugated with allophycocyanin (cat no. 103011, BioLegend) were used. The cells treated with the agents for $24 \mathrm{~h}$ were trypsinized and washed 3 times with PBS. The cells $\left(1 \times 10^{6}\right)$ in a single-cell suspension were resuspended in the staining buffer (PBS containing 2\% FBS) and labeled with the antibodies, followed by washing and resuspension in $500 \mu 1$ of staining buffer according to the manufacturer's instructions. The cells were also labeled with propidium iodide to enrich viable cells and analyzed with a JSAN cell sorter and AppSan software (Bay Bioscience, Kobe, Japan).

Enrichment of CD24-positive cells. To obtain a cell population enriched in CD24-positive cells, a magnetic cell-sorting system with the Miltenyi Biotec MACS Cell Separation kit
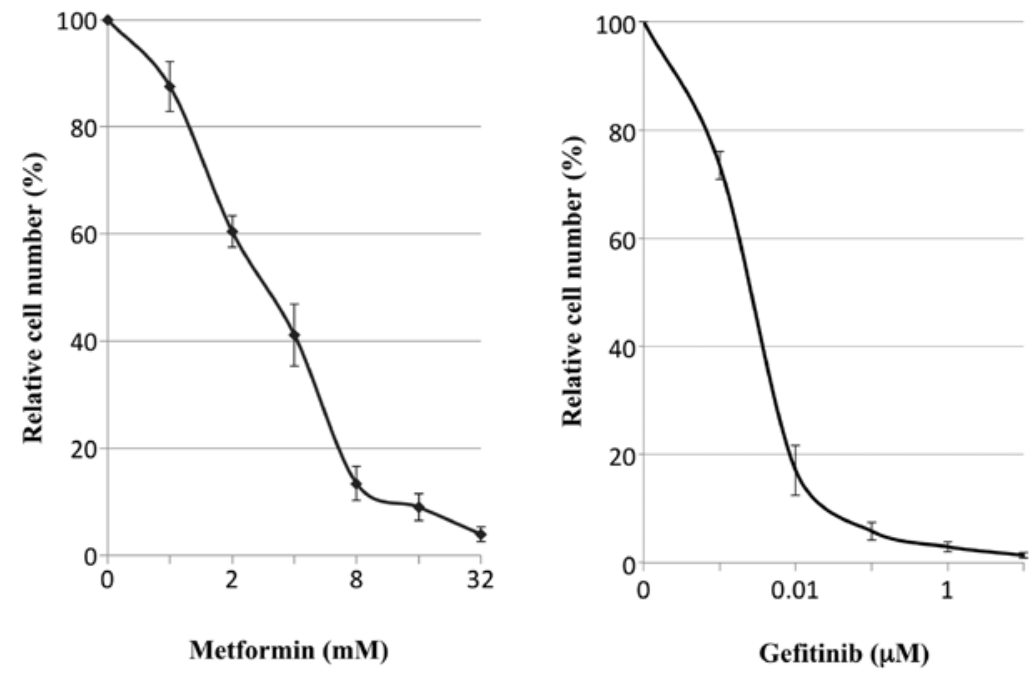

Figure 2. Dose-dependent growth inhibition of PC9 cells with metformin or gefitinib in vitro. The dots and bars represent the mean and SE (n=3), respectively. 


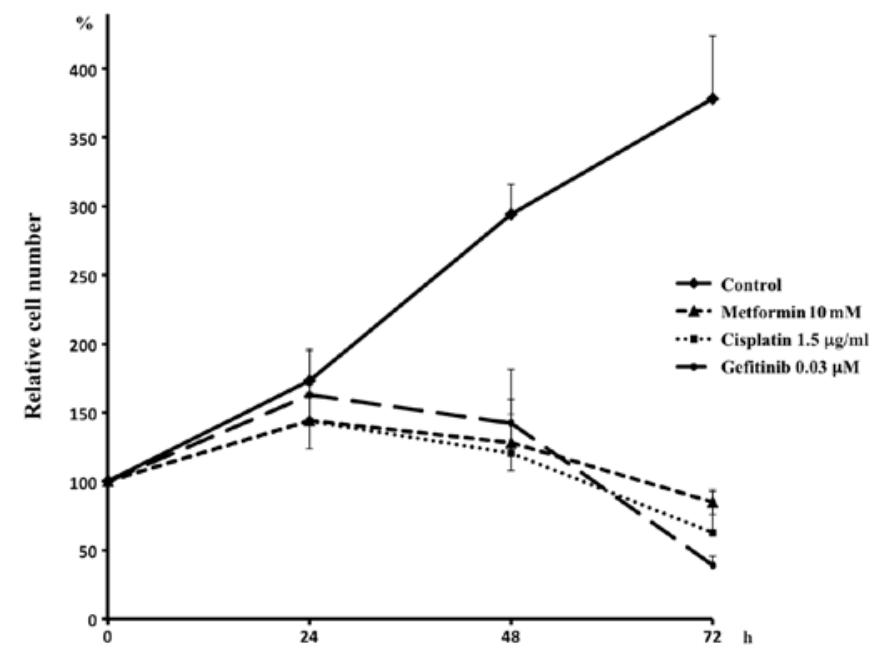

Figure 3. Growth curves of PC9 cells in vitro. In contrast to the cells that grew to $380 \%$ of the originally plated cell number without any agent after $72 \mathrm{~h}$ of culture, metformin, gefitinib and cisplatin suppressed cell growth Because the concentrations of the agents adopted here were almost equivalent in suppression, they were used for further experiments. The dots and bars represent the mean and $\mathrm{SE}(\mathrm{n}=3)$, respectively.

was used according to the manufacturer's instructions. The sorted cells were analyzed with a JSAN cell sorter and the AppSan software as described above. The cell sorting and subsequent brief culture for propagation were repeated up to 4 times to obtain a cell population consisting of $\sim 80 \%$ CD24positive cells.

Statistical analysis. Data are presented as the means \pm SEs as indicated and were analyzed by Student's t-test. A p-value of $<0.05$ (2-tailed) was considered statistically significant.

\section{Results}

Effect of gefitinib and metformin on xenograft tumors in vivo. When the administration was initiated day 16 or when the tumor size reached $\sim 300 \mathrm{~mm}^{3}$, metformin did not reduce tumor growth (compare groups 2 to 1, Fig. 1). In addition, no additional effect was observed with metformin in tumor shrinkage with gefitinib (see the curves from days 16 to 31 in groups 3 and 4). Metformin, however, significantly reduced tumor regrowth after withdrawal of gefitinib treatment (see the curves from days 35 to 66 in groups 3 and 4).

Suppression of in vitro cell proliferation. In vitro administration of metformin suppressed proliferation of PC9 cells in a dose-dependent manner, similarly to gefitinib (Fig. 2) and the concentrations that resulted in $90 \%$ reduction of cell numbers compared with controls at $72 \mathrm{~h}$ after the administration were $11.6 \pm 1.87 \mathrm{mM}$ with metformin, $0.042 \pm 0.024 \mu \mathrm{M}$ with gefitinib and $1.48 \pm 0.18 \mu \mathrm{g} / \mathrm{ml}$ with cisplatin. In subsequent experiments, concentrations of $10 \mathrm{mM}$ for metformin, $0.03 \mu \mathrm{M}$ for gefitinib and $1.5 \mu \mathrm{g} / \mathrm{ml}$ for cisplatin were employed (Fig. 3).

Apoptosis induction by the agents. As assessed by Hoechst staining, metformin did not induce more apoptotic cells compared with the control in contrast to cisplatin and gefitinib (Fig. 4A). The representative morphology in the presence of the agents is shown in Fig. 5. The caspase 3 assay confirmed the results (Fig. 4B) with statistically significant differences, although the caspase 8 assay solely revealed a trend without statistical significance (Fig. 4C).

Cell cycle alteration. FACS analysis showed that metformin did not significantly alter the cell cycle distribution compared to the control. In contrast, cisplatin caused a marked cell accu-
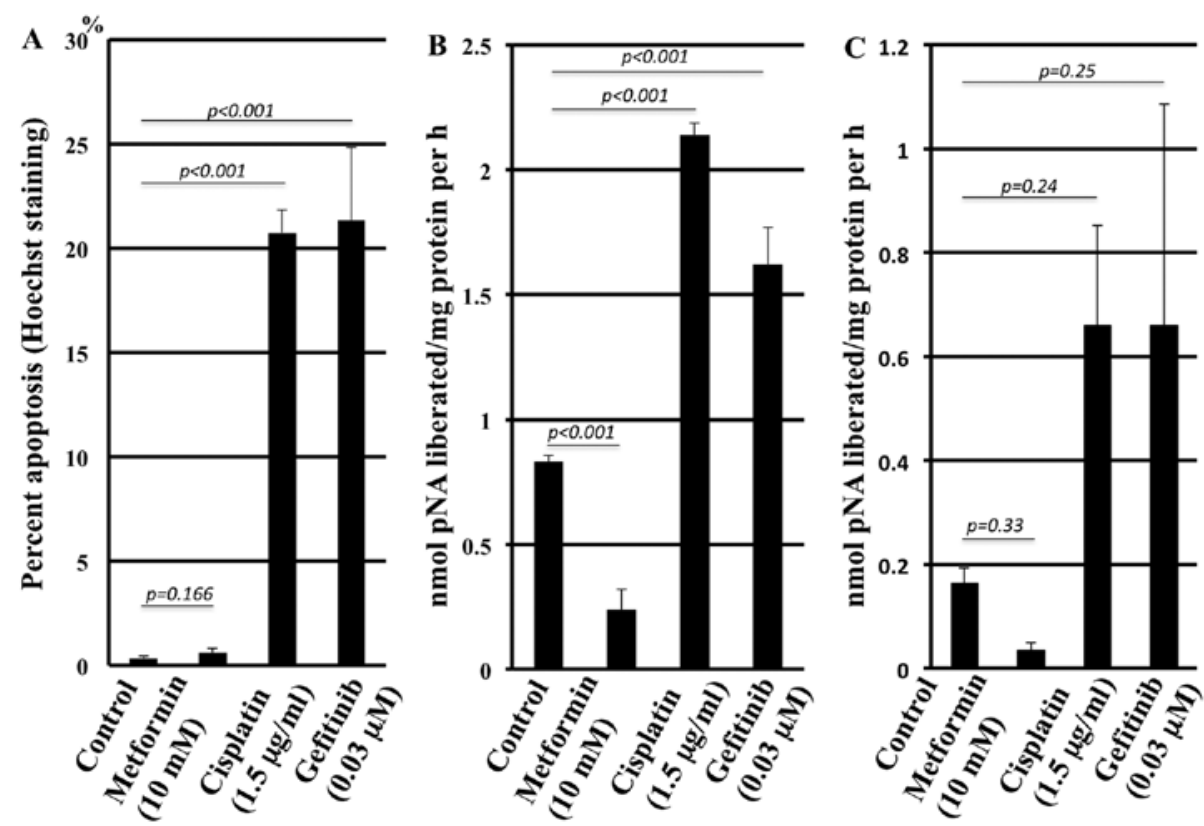

Figure 4. Apoptotic cells were counted by Hoechst staining $48 \mathrm{~h}$ after exposure to the agents. Percentage of apoptotic cells per total cells was determined (A). Apoptosis was also assessed by caspase 3 (B) and caspase 8 (C) activities, $24 \mathrm{~h}$ after exposure to the agents. The columns and bars represent the mean and SE $(\mathrm{n}=3)$, respectively. 
A

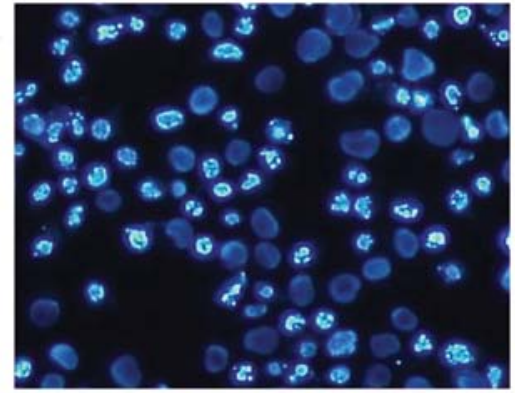

B

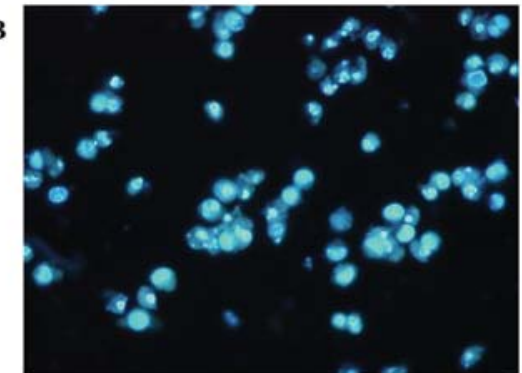

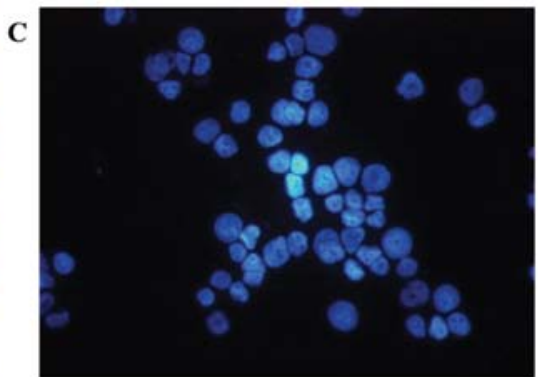

Figure 5. Apoptotic cells were counted by Hoechst staining $48 \mathrm{~h}$ after exposure to the agents. Representative morphology in the presence of cisplatin (A), gefitinib (B) and metformin (C) is shown. Metformin failed to induce apoptosis in contrast to cisplatin and gefitinib.
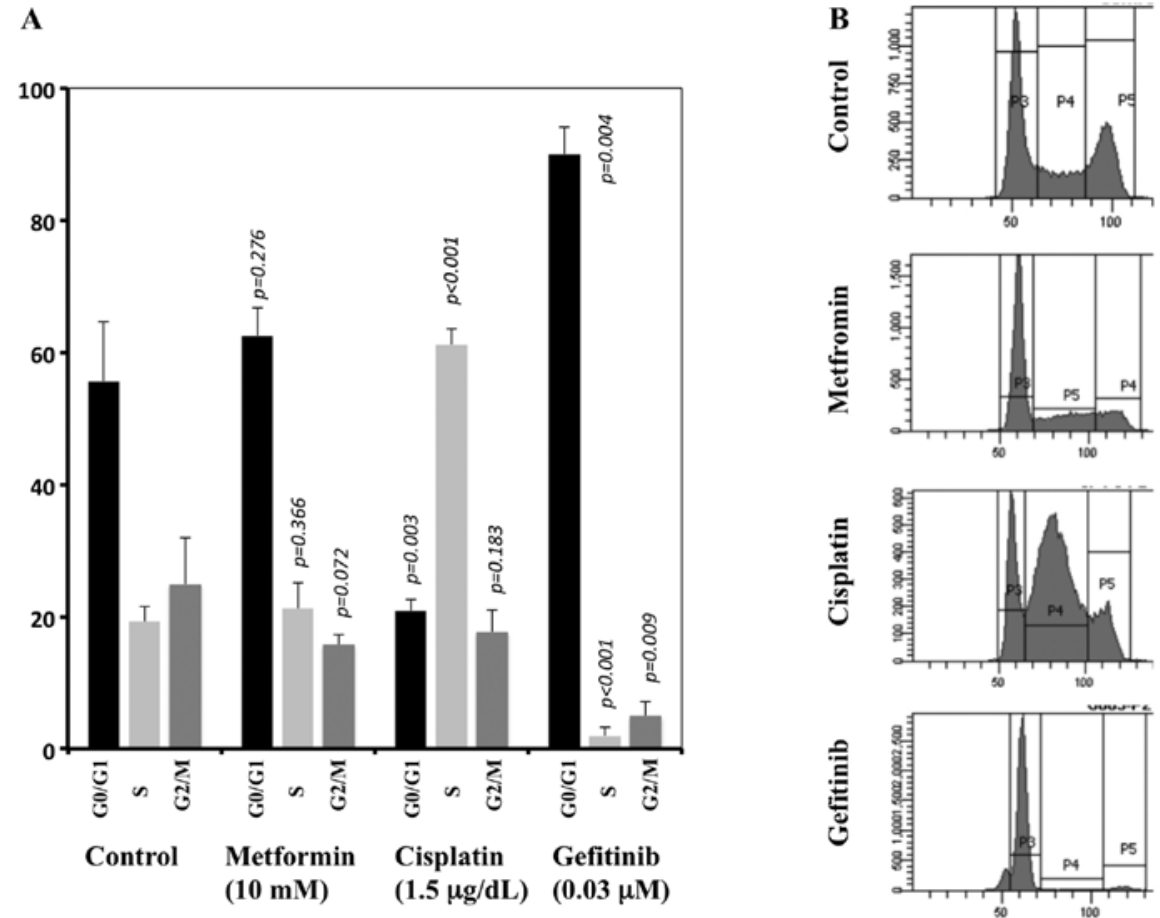

Figure 6. Altered cell cycle distributions $24 \mathrm{~h}$ after exposure to the agents, assessed by FACS. The cell cycle distribution was not significantly altered with metformin, in contrast with cisplatin and gefitinib. The columns and bars represent the mean and SE [n=3, (A)], respectively. The p-value for difference between each column and the corresponding column in the control is given. A representative FACS analysis with each agent is shown (B).

mulation at the $\mathrm{S}$ phase together with a marked decrease of cells at the G0/G1 phases. Gefitinib caused marked accumulation at the G0/G1 phases together with a marked decrease at the $\mathrm{S}$ and $\mathrm{G} 2 / \mathrm{M}$ phases compared with the control (Fig. 6).

Expression of CD24, CD44 and CD133 in surviving cells after exposure to gefitinib. Because we failed to detect reproducible CD133 expression by FACS analysis, CD133 expression was assessed solely by immunofluorescence staining. The CD133- positive cells were significantly enriched after exposure to gefitinib for $24 \mathrm{~h}$. In contrast, exposure to metformin alone or to gefitinib combined with metformin did not augment CD133 expression (Fig. 7). Altered expression of CD24 and CD44, assessed by FACS, are shown in Fig. 8. The cell population with negative staining for CD44, either with or without CD24 expression, was not significantly altered by exposure to gefitinib for $24 \mathrm{~h}$. The cell population with positive staining for CD24 increased significantly after exposure to gefitinib for $24 \mathrm{~h}$. 


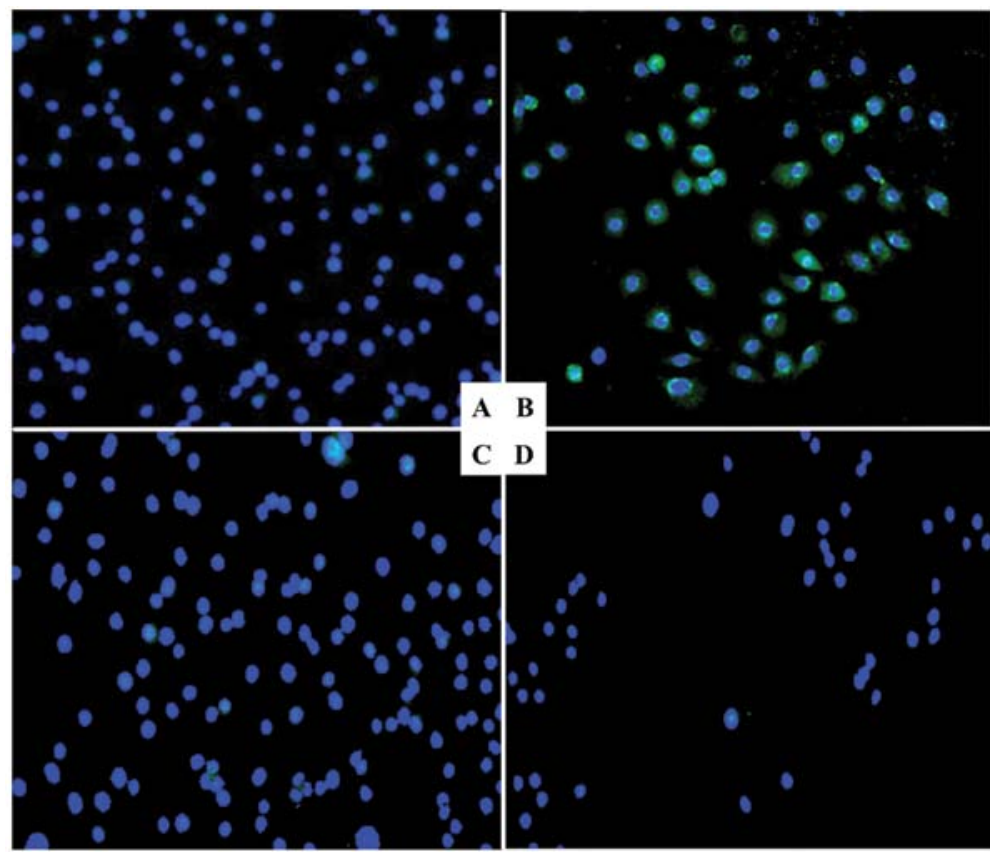

Figure 7. Immunofluorescent staining of CD133 in cells. Compared with the untreated cells (A), the population treated by gefitinib was enriched with CD133 positive cells (B). In contrast, metformin treatment did not enrich CD133 positive cells (C). When the cells were treated with both agents, no enrichment was observed (D).

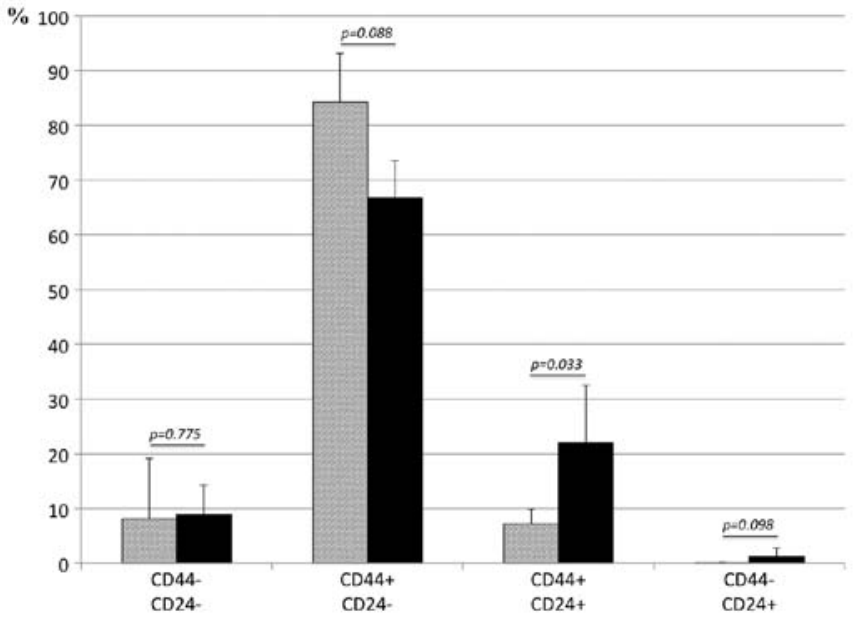

Figure 8. FACS analysis for altered expression of CD24 and CD44 after gefitinib treatment. The shaded and closed columns represent the mean percentages of the untreated and treated cells, respectively. The bars represent the SE ( $n=3)$. CD24 expressing cells significantly increased during gefitinib treatment, from $\sim 8-23 \%$, with CD44 expressing cells unaltered.

Sensitivity to metformin and gefitinib in CD24-positive cells. To obtain a cell population enriched in CD24-positive cells, the parental cells were sorted by FACS magnetic separation. Because a single sorting was not sufficient to enrich CD24-positive cells, the sorting was repeated up to 4 times. The proportion of CD24-positive cells in the parental cells $(7.1 \pm 4.2 \%, \mathrm{n}=3)$ was enriched to $81.8 \pm 12.1 \%(\mathrm{n}=3)$. The in vitro sensitivity assay revealed that the parental and sorted cells showed nearly identical sensitivity to metformin. The sorted cells, however, were slightly but significantly more resistant to gefitinib than the parental cells (Fig. 9).

\section{Discussion}

The present study employed the human lung adenocarcinoma cell line PC9, which possesses an EGFR exon 19 deletion mutation that renders EGFR sensitive to the TKIs. In vivo experiments with xenografts derived from these cells resulted in the following observations: i) metformin exerted no effect on already grown tumors $\left(>300 \mathrm{~mm}^{3}\right)$, ii) metformin had no additional effect on tumor shrinkage by gefitinib, iii) the tumors regrew after withdrawing gefitinib even after the treatment had resulted in complete regression of the tumors and iv) metformin significantly suppressed the regrowth of the tumors after withdrawing gefitinib treatment. These observations suggest that metformin is effective specifically on residual cells after gefitinib treatment; however, metformin is not sufficiently effective to suppress growth of already established tumors.

To test our hypothesis, a series of in vitro experiments were conducted. Cisplatin was included as a positive control in some of the experiments. A dose that resulted in an in vitro cell number reduction to $10 \%$ of the original cell number was chosen for each agent. Metformin did not induce apoptosis when assessed by Hoechst staining and caspase 3 and 8 activity determination, in contrast to gefitinib and cisplatin. Moreover, apoptosis induction by metformin treatment was significantly lower, even lower than what was observed in the control experiments, suggesting an apoptosis-protective property of metformin. This is consistent with a previous report that demonstrated a preventive effect of AMPK on apoptosis $(33,34)$. Although metformin decreased the cells at the $\mathrm{G} 2 / \mathrm{M}$ phases, cell cycle alteration with metformin was not dramatic in contrast with cisplatin and gefitinib, which induced significant accumulation at the $\mathrm{S}$ and $\mathrm{G} 0 / \mathrm{G} 1$ phases, 


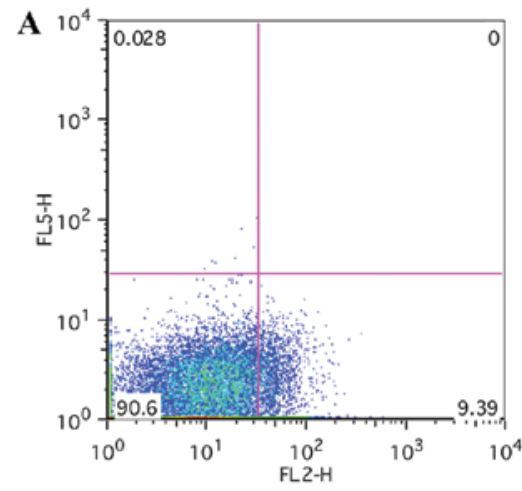

C
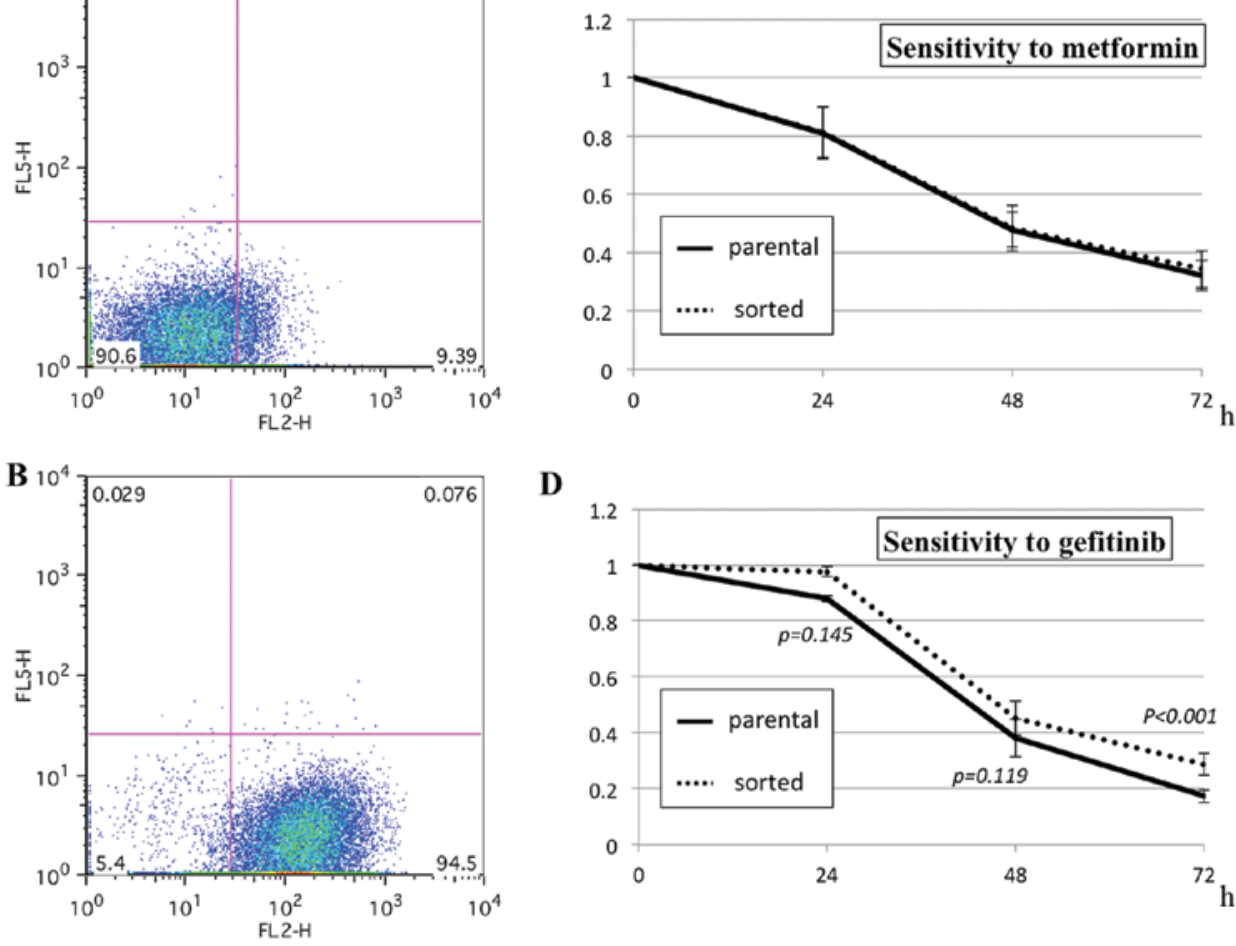

D

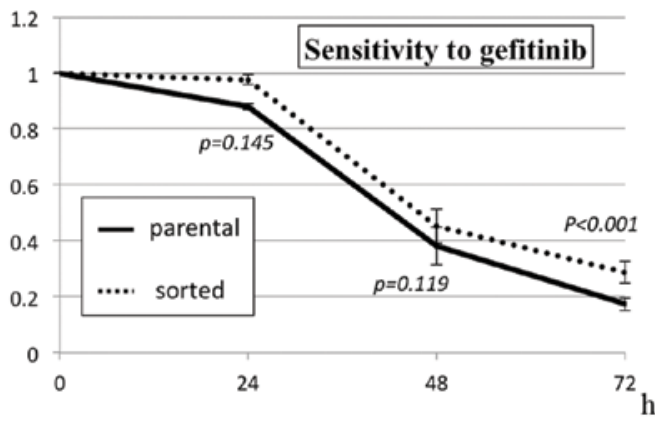

Figure 9. The proportion of CD24-positive cells [the right lower squares in (A and B)] increased from 7.1 $\pm 4.2 \%(\mathrm{n}=3)$ in the parental cells $(\mathrm{A})$ to $81.8 \pm 12.1 \%$ $(\mathrm{n}=3)$ in the sorted cells (B). The sensitivity to metformin (C) and gefitinib (D) of these cells were compared in vitro.

respectively. Although the results obtained with cisplatin (35) and gefitinib $(36,37)$ are consistent with previously reported data, the results obtained with metformin are rather complicated. Some studies reported an absence of apoptosis induction with metformin (17), whereas other reports described a significant apoptosis induction (12). Similarly, although some have reported a significant cell cycle shift with metformin $(12,16)$, others have reported only mild cell cycle arrest at the G0-G1 phases in the presence of metformin $(38,39)$. The effects of metformin on apoptosis and cell cycle arrest may vary depending on the cell line examined, as previously reported with human lung cancers of a variety of histological types (40). Nevertheless, the minimum effects of metformin on apoptosis induction and cell cycle alteration in vitro in the present experimental system may explain the absence of effects on tumor growth inhibition in vivo.

Based on the reported drug resistance of cancer stem cells (41) and the information presented in a previous study (5), the expression of 3 putative cancer stem cell markers, CD133 (42), CD44 (43) and CD24 (44), was examined. After gefitinib treatment of the cells in vitro, cells with CD133 expression were enriched as assessed by immunofluorescence staining. FACS analysis revealed an enrichment of cells with CD24 expression after gefitinib treatment in vitro. The population of cells with CD44 expression was unaltered. These observations are similar to those in a previous report (5), suggesting that cells with CD133 or CD24 expression may be resistant to gefitinib. In fact, metformin treatment in vitro did not enrich cells with CD133 expression, in contrast to what was observed with gefitinib. In addition, combined treatment with metformin and gefitinib canceled the enrich- ment observed in the treatment with gefitinib alone. These results strongly suggest that metformin is effective against residual cells after in vitro treatment with gefitinib, consistent with the in vivo experiment. A cell population consisting of $\sim 80 \%$ cells expressing CD24 ( 10-fold enrichment compared with the parental cells) was obtained by FACS sorting to directly examine chemosensitivity. These cells were slightly but significantly more resistant to gefitinib than the parental cells, whereas their sensitivity to metformin was identical to the parental cells, suggesting that metformin was effective against residual cells after gefitinib treatment. Nevertheless, the degree of augmented resistance to gefitinib in cells with CD24 expression was unexpectedly small. This can be explained if the resistant cells express CD24 and if only a part of the cell population with CD24 expression is resistant to gefitinib.

The nature and properties of the residual cells after treatment with gefitinib are unclear. Although CD133 and CD24 are putative markers for cancer stem cells in human brain and colon cancers, respectively, the present results do not indicate that the residual cells are cancer stem cells because cancer stem cells in human lung cancer have not yet been identified. Considering their prompt emergence in a short period, non-mutational mechanisms, including epigenetic change and selection of resistant cells from a heterogeneous cell population, seem to be the most likely routes of chemoresistance. Cancer stem cells and epithelial-mesenchymal transitions might be possible candidates for selection. Specific targeting of residual cells after chemotherapy may be a suitable approach to cure cancers. The present study highlighted metformin as a candidate for targeting residual 
cells and we envision that further elucidation of the detailed molecular mechanism of the cytotoxicity of metformin represents progress in cancer therapy.

\section{Acknowledgements}

Yuichi Takiguchi has received honoraria for lectures not related to this study and unrestricted research and educational grants from AstraZeneca, K.K., Japan, and Chugai Pharmaceutical Co., Japan. Ikuo Sekine and Koichiro Tatsumi have received honoraria for lectures not related to this studyfrom AstraZeneca, K.K., Japan, and Chugai Pharmaceutical Co., Japan. Osamu Yokosuka has received research grants not related to this study from Chugai Pharmaceutical Co., Japan. This study was supported by a Grant-in-Aid for Scientific Research (grant no. 23591136) from the Japan Society for the Promotion of Science and the Ministry of Education, Culture, Sports, Science and Technology of Japan.

\section{References}

1. Inoue A, Kobayashi K, Maemondo M, et al: Updated overall survival results from a randomized phase III trial comparing gefitinib with carboplatin-paclitaxel for chemo-naive non-small cell lung cancer with sensitive EGFR gene mutations (NEJ002). Ann Oncol 24: 54-59, 2012.

2. Wakeling AE, Guy SP, Woodburn JR, et al: ZD1839 (Iressa): an orally active inhibitor of epidermal growth factor signaling with potential for cancer therapy. Cancer Res 62: 5749-5754, 2002 .

3. Kosaka T, Yatabe Y, Endoh H, et al: Analysis of epidermal growth factor receptor gene mutation in patients with non-smal cell lung cancer and acquired resistance to gefitinib. Clin Cancer Res 12: 5764-5769, 2006.

4. Engelman JA, Zejnullahu K, Mitsudomi T, et al: MET amplification leads to gefitinib resistance in lung cancer by activating ERBB3 signaling. Science 316: 1039-1043, 2007.

5. Sharma SV, Lee DY, Li B, et al: A chromatin-mediated reversible drug-tolerant state in cancer cell subpopulations. Cell 141: 69-80, 2010.

6. Towler MC and Hardie DG: AMP-activated protein kinase in metabolic control and insulin signaling. Circ Res 100: 328-341, 2007.

7. Pollak MN: Investigating metformin for cancer prevention and treatment: the end of the beginning. Cancer Discov 2: 778-790, 2012.

8. Evans JM, Donnelly LA, Emslie-Smith AM, Alessi DR and Morris AD: Metformin and reduced risk of cancer in diabetic patients. BMJ 330: 1304-1305, 2005.

9. Bowker SL, Majumdar SR, Veugelers P and Johnson JA: Increased cancer-related mortality for patients with type 2 diabetes who use sulfonylureas or insulin. Diabetes Care 29: 254-258, 2006.

10. Jiralerspong S, Palla SL, Giordano SH, et al: Metformin and pathologic complete responses to neoadjuvant chemotherapy in diabetic patients with breast cancer. J Clin Oncol 27: 3297-3302, 2009.

11. Mazzone PJ, Rai H, Beukemann M,Xu M, Jain A and Sasidhar M: The effect of metformin and thiazolidinedione use on lung cancer in diabetics. BMC Cancer 12: 410, 2012.

12. Alimova IN, Liu B, Fan Z, et al: Metformin inhibits breast cancer cell growth, colony formation and induces cell cycle arrest in vitro. Cell Cycle 8: 909-915, 2009.

13. Liu B, Fan Z, Edgerton SM, et al: Metformin induces unique biological and molecular responses in triple negative breast cancer cells. Cell Cycle 8: 2031-2040, 2009.

14. Zakikhani M, Dowling R, Fantus IG, Sonenberg N and Pollak M: Metformin is an AMP kinase-dependent growth inhibitor for breast cancer cells. Cancer Res 66: 10269-10273, 2006.

15. Rocha GZ, Dias MM, Ropelle ER, et al: Metformin amplifies chemotherapy-induced AMPK activation and antitumoral growth. Clin Cancer Res 17: 3993-4005, 2011.
16. Ben Sahra I, Laurent K, Loubat A, et al: The antidiabetic drug metformin exerts an antitumoral effect in vitro and in vivo through a decrease of cyclin D1 level. Oncogene 27: 3576-3586, 2008.

17. Wang LW, Li ZS, Zou DW, Jin ZD, Gao J and Xu GM: Metformin induces apoptosis of pancreatic cancer cells. World J Gastroenterol 14: 7192-7198, 2008.

18. Gotlieb WH, Saumet J, Beauchamp MC, et al: In vitro metformin anti-neoplastic activity in epithelial ovarian cancer. Gynecol Oncol 110: 246-250, 2008.

19. Rattan R, Giri S, Hartmann LC and Shridhar V: Metformin attenuates ovarian cancer cell growth in an AMP-kinase dispensable manner. J Cell Mol Med 15: 166-178, 2011.

20. Rattan R, Graham RP, Maguire JL, Giri S and Shridhar V: Metformin suppresses ovarian cancer growth and metastasis with enhancement of cisplatin cytotoxicity in vivo. Neoplasia 13: 483-491, 2011.

21. Hawley SA, Boudeau J, Reid JL, et al: Complexes between the LKB1 tumor suppressor, STRAD alpha/beta and MO25 alpha/ beta are upstream kinases in the AMP-activated protein kinase cascade. J Biol 2: 28, 2003.

22. Lizcano JM, Goransson O, Toth R, et al: LKB1 is a master kinase that activates 13 kinases of the AMPK subfamily, including MARK/PAR-1. EMBO J 23: 833-843, 2004.

23. Dowling RJ, Zakikhani M, Fantus IG, Pollak M and Sonenberg N: Metformin inhibits mammalian target of rapamycin-dependent translation initiation in breast cancer cells. Cancer Res 67: 10804-10812, 2007.

24. Bolster DR, Crozier SJ, Kimball SR and Jefferson LS: AMP-activated protein kinase suppresses protein synthesis in rat skeletal muscle through down-regulated mammalian target of rapamycin (mTOR) signaling. J Biol Chem 277: 23977-23980, 2002.

25. Kimura N, Tokunaga C, Dalal S, et al: A possible linkage between AMP-activated protein kinase (AMPK) and mammalian target of rapamycin (mTOR) signalling pathway. Genes Cells 8: 65-79, 2003.

26. Inoki K, Zhu T and Guan KL: TSC 2 mediates cellular energy response to control cell growth and survival. Cell 115: 577-590, 2003.

27. Iliopoulos D, Hirsch HA and Struhl K: Metformin decreases the dose of chemotherapy for prolonging tumor remission in mouse xenografts involving multiple cancer cell types. Cancer Res 71: 3196-3201, 2011.

28. Janjetovic K, Vucicevic L, Misirkic M, et al: Metformin reduces cisplatin-mediated apoptotic death of cancer cells through AMPK-independent activation of Akt. Eur J Pharmacol 651: 41-50, 2011.

29. Harhaji-Trajkovic L, Vilimanovich U, Kravic-Stevovic T, Bumbasirevic V and Trajkovic V: AMPK-mediated autophagy inhibits apoptosis in cisplatin-treated tumour cells. J Cell Mol Med 13: 3644-3654, 2009.

30. Costa DB, Halmos B, Kumar A, et al: BIM mediates EGFR tyrosine kinase inhibitor-induced apoptosis in lung cancers with oncogenic EGFR mutations. PLoS Med 4: 1669-1679, 2007.

31. Memmott RM, Mercado JR, Maier CR, Kawabata S, Fox SD and Dennis PA: Metformin prevents tobacco carcinogen-induced lung tumorigenesis. Cancer Prev Res 3: 1066-1076, 2010.

32. Sirotnak FM, Zakowski MF, Miller VA, Scher HI and Kris MG Efficacy of cytotoxic agents against human tumor xenografts is markedly enhanced by coadministration of ZD1839 (Iressa), an inhibitor of EGFR tyrosine kinase. Clin Cancer Res 6: 4885-4892, 2000.

33. Blazquez C, Geelen MJ, Velasco G and Guzman M: The AMP-activated protein kinase prevents ceramide synthesis de novo and apoptosis in astrocytes. FEBS Lett 489: 149-153, 2001.

34. Shaw RJ, Kosmatka M, Bardeesy N, et al: The tumor suppressor LKB1 kinase directly activates AMP-activated kinase and regulates apoptosis in response to energy stress. Proc Natl Acad Sci USA 101: 3329-3335, 2004

35. Ormerod MG, Orr RM and Peacock JH: The role of apoptosis in cell killing by cisplatin: a flow cytometric study. Br J Cancer 69: 93-100, 1994

36. Janmaat ML, Kruyt FA, Rodriguez JA and Giaccone G: Response to epidermal growth factor receptor inhibitors in non-small cell lung cancer cells: limited antiproliferative effects and absence of apoptosis associated with persistent activity of extracellular signal-regulated kinase or Akt kinase pathways. Clin Cancer Res 9: 2316-2326, 2003. 
37. Tracy S, Mukohara T, Hansen M, Meyerson M, Johnson BE and Janne PA: Gefitinib induces apoptosis in the EGFRL858R non-small-cell lung cancer cell line H3255. Cancer Res 64: 7241-7244, 2004.

38. Cantrell LA, Zhou C, Mendivil A, Malloy KM, Gehrig PA and Bae-Jump VL: Metformin is a potent inhibitor of endometrial cancer cell proliferation - implications for a novel treatment strategy. Gynecol Oncol 116: 92-98, 2010.

39. Kato K, Gong J, Iwama $\mathrm{H}$, et al: The antidiabetic drug metformin inhibits gastric cancer cell proliferation in vitro and in vivo. Mol Cancer Ther 11: 549-560, 2012.

40. Ashinuma H, Takiguchi Y, Kitazono S, et al: Antiproliferative action of metformin in human lung cancer cell lines. Oncol Rep 28: 8-14, 2012.
41. Matsui W, Wang Q, Barber JP, et al: Clonogenic multiple myeloma progenitors, stem cell properties, and drug resistance. Cancer Res 68: 190-197, 2008.

42. Hemmati HD, Nakano I, Lazareff JA, et al: Cancerous stem cells can arise from pediatric brain tumors. Proc Natl Acad Sci USA 100: 15178-15183, 2003.

43. Al-Hajj M, Wicha MS, Benito-Hernandez A, Morrison SJ and Clarke MF: Prospective identification of tumorigenic breast cancer cells. Proc Natl Acad Sci USA 100: 3983-3988, 2003.

44. Vermeulen L, Todaro M, de Sousa Mello F, et al: Single-cell cloning of colon cancer stem cells reveals a multi-lineage differentiation capacity. Proc Natl Acad Sci USA 105: 13427-13432, 2008. 